\title{
Treatment of Acromegaly \\ Increases BMD but Reduces Trabecular Bone Score - a longitudinal study
}

\author{
Kristin Godang ${ }^{1}$, Nicoleta Cristina Olarescu ${ }^{1}$, Jens Bollerslev,2 Ansgar Heck ${ }^{1,2}$ \\ ${ }^{1}$ Section for Specialized Endocrinology; Department of Endocrinology, Oslo University Hospital Rikshospitalet, \\ ${ }^{2}$ Faculty of Medicine, University of Oslo, Norway
}

\begin{abstract}
Background
Dual Energy X-ray Absorptiometry (DXA) is the primary screening tool for assessing bone mass in osteoporosis, but does not predict the increased vertebral fracture risk in acromegaly [1]. Bone mineral density (BMD) alone does not provide information regarding the structural characteristics of bone. This limitation has been a driving force in the search for a new and noninvasive technology reflecting cancellous bone microarchitecture, namely Trabecular Bone Score (TBS) [2, 3].
\end{abstract}

\section{Aims}

1) To study the longitudinal changes of vertebral trabecular bone microarchitecture as measured by TBS and site-matched BMD before and following treatment of acromegaly.

2) To study the BMD changes in other compartments assessed by DXA and thereby enhance the understanding of bone physiology in acromegaly by correlations to biochemical markers of disease activity and bone turnover.

\section{Methods}

- 38 patients with acromegaly consecutively recruited between 2005-2015.

- DXA scans and blood samples for measuring bone turnover (PINP, CTX1) were acquired at baseline and one year after transsphenoidal surgery.

- TBS was analysed at L1-L4 (LS) with TBS iNsight software.

\section{Results}

Following treatment, mean TBS decreased by $3.0( \pm 7.0) \%$, whereas BMD at the lumbar spine (LS; L1-L4) increased by $3.2( \pm 4.9) \%$ (both $p<0.01)$ (Table 1$)$. The changes in BMD LS and TBS were not correlated ( $p=0.87)$. The decrease of TBS and the increase of BMD was observed just in men (Figure 1).

$B M D$ increased in the ultradistal radius and total body (both $\mathrm{p}<0.01$ ) (Table 1) The increase in BMD (LS) was associated with a decrease in P1NP and CTX-1 $(p<0.001)$ and with lower levels of P1NP and CTX-1 at follow-up $(p<0.02)$. The decrease of disease activity, as estimated by IGF-1, was positively associated with the decrease of bone markers (Figure 2)

\section{Discussion}

We found that treatment for acromegaly resulted in an increase of BMD LS, but decrease of TBS. This effect was driven by male and eugonadal patients.

The reduction of bone turnover markers predicted the increase in BMD, but not the decrease in TBS.

Previous studies addressing the issue of skeletal complications in acromegaly have demonstrated an increased risk for vertebral fractures despite a relatively normal BMD by DXA [1]. TBS has been demonstrated to be decreased in a crosssectional study [4]. Our study gives a broad longitudinal description of the TBS (LS) and BMD changes in different compartments, and increases insight in mechanisms potentially involved in cancellous bone microarchitecture changes and fracture risk in acromegaly.

The study underscores the limitations of BMD as a screening tool for secondary osteoporosis and adds important knowledge about the reduced bone quality in acromegaly.

\section{References}

[1] Claessen KM et al. Progression of vertebral fractures despite long-term biochemical control of acromegaly: a prospective follow-up study. JCEM 201398 p.4808 [2] Hans D et al. Correlations between trabecula bo pospen dimensional parane, me bon Clin Densitom 201114 p.302 [3] Winzenrieth R et al. Three-dimensional (3D) microarchitecture correlations with $2 \mathrm{D}$ projection image gray-level variations assessed by trabecular bone score using high-resolution computed tomographic acquisitions: effects of resolution and noise. J Clin Densitom 201316 p287. [4] Hong AR, et al. Trabecular bone score as a skeletal fragility index in acromegaly patients. Osteoporosis international, 2016; 27:1123

\section{Conflict of interest}

JB has received unrestricted research grants from Novartis, Pfizer and Merck Norway AS. AH has received speaker fees from Novartis, Ipsen, NovoNordisk, Pfizer and participated in Novartis' Nordic Advisory Board.
Table 1: Longitudinal measurements at baseline and 1 year postoperatively

\begin{tabular}{|c|c|c|c|}
\hline $\mathrm{n}=38$; (female 14; male 24) & Baseline & 1yr PO visit & $\mathbf{P}$ \\
\hline Age & $49.0(14.1)$ & n.a. & \\
\hline $\mathrm{BMI}\left(\mathrm{kg} / \mathrm{m}^{2}\right)$ & $28.7(4.2)$ & $28.6(4.9)$ & \\
\hline \multicolumn{4}{|l|}{ DXA } \\
\hline TBS LS & $1.331(0.147)$ & $1.288(0.137)$ & $<0.001$ \\
\hline BMD LS & $1.204(0.194)$ & $1.241(0.199)$ & $<0.001$ \\
\hline BMD total hip & $1.044(0.148)$ & $1.054(0.137)$ & .059 \\
\hline BMD femoral neck & $0.994(0.146)$ & $0.992(0.146)$ & .896 \\
\hline BMD proximal radius & $0.880(0.138)$ & $0.894(0.138)$ & .099 \\
\hline BMD ultradistal radius & $0.478(0.092)$ & $0.514(0.097)$ & $<0.001$ \\
\hline BMD total body & $1.184(0.163)$ & $1.228(0.177)$ & $<0.001$ \\
\hline $25(\mathrm{OH}) \mathrm{D}(\mathrm{nmol} / \mathrm{l})$ & $47(19)$ & $51(21)$ & .360 \\
\hline P1NP $(\mu g / l)$ & $73(56,136)$ & $27(17,36)$ & $<0.001$ \\
\hline CTX-1 $(\mu \mathrm{g} / \mathrm{I})$ & $0.86(0.60,1.79)$ & $0.36(0.22,0.54)$ & $<0.001$ \\
\hline IGF-1 (nmol/I) & $109.4(43.1)$ & $35.2(19.2)$ & $<0.001$ \\
\hline IGF-1/ULN & $2.8(1.6,3.5)$ & $0.8(0.6,1.3)$ & $<0.001$ \\
\hline $\mathrm{GH}(\mu \mathrm{g} / \mathrm{l})$ & $6.7(3.1,15.8)$ & $0.9(0.4,2.7)$ & $<0.001$ \\
\hline
\end{tabular}

Data is presented as mean $( \pm S D)$ or median $(25,75$ percentile). $P$ value assessed by paired sample T-test or Wilcoxon signed rank test.

Figure 1: Gender difference in the change in TBS and BMD LS
TBS

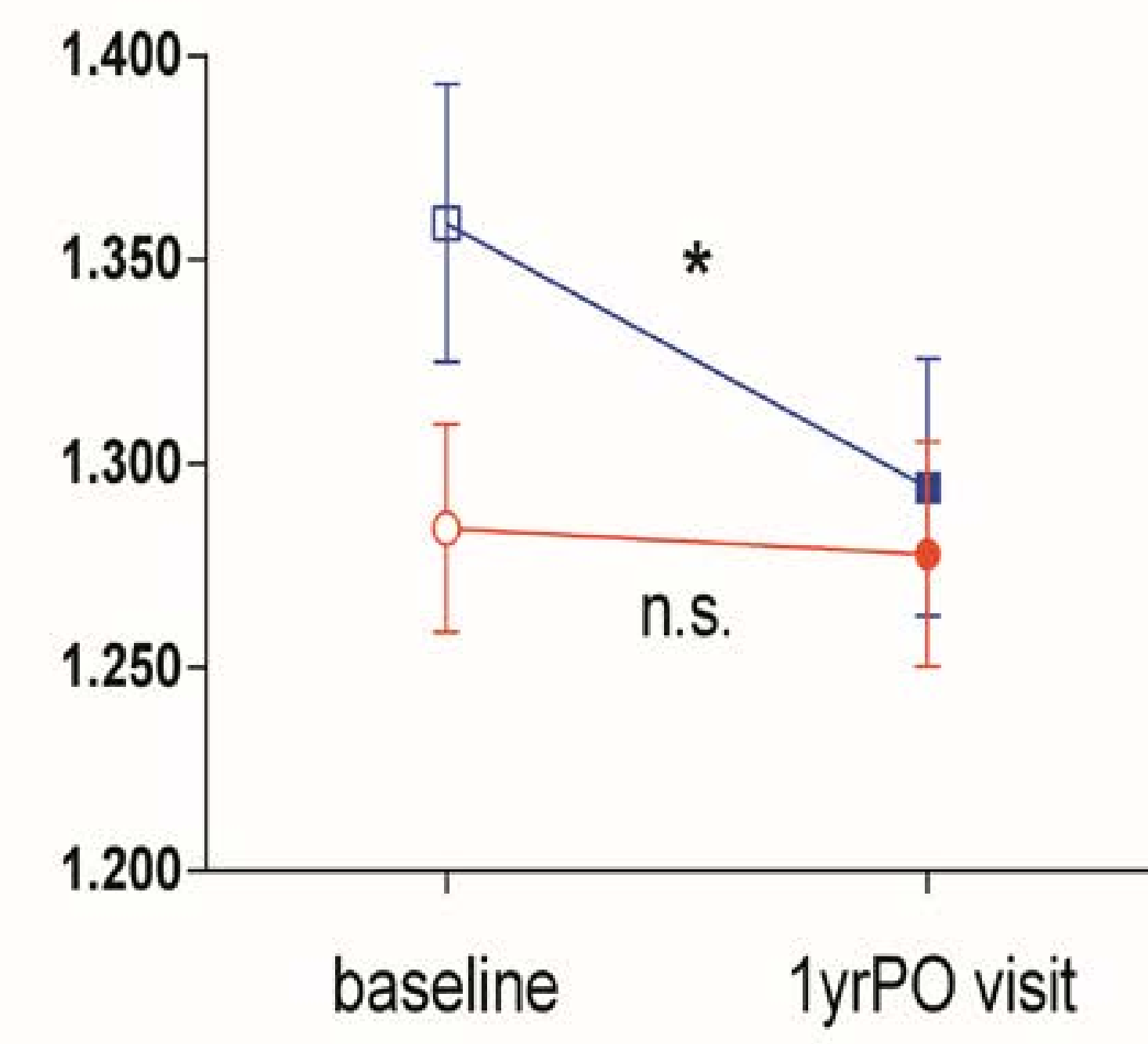

BMD

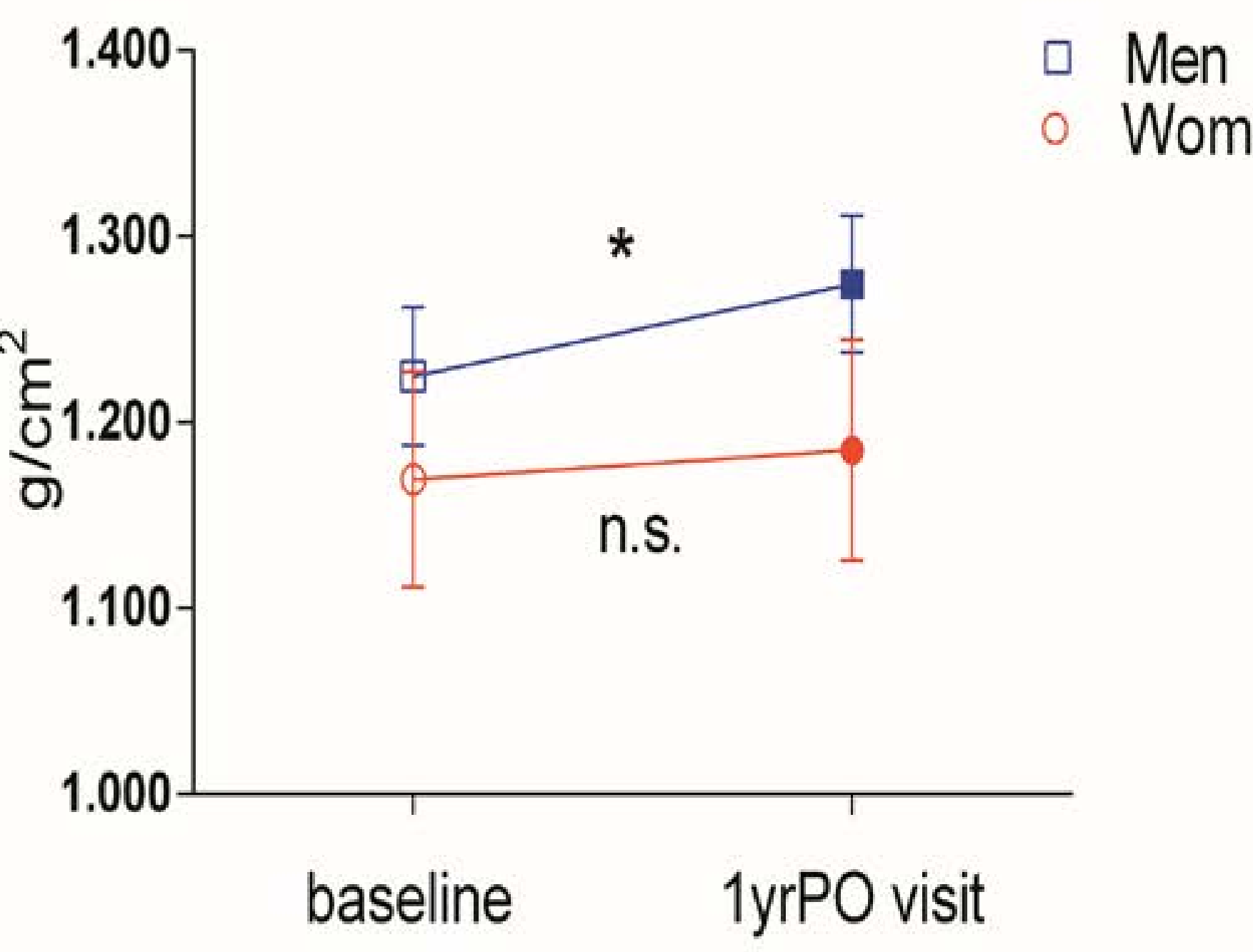

TBS change in men was $-4.5 \%( \pm 6.7 ; p=0.003)$ and in women $-0.3 \%( \pm 6.8 ; p=0.85)$ ( $p=0.063$ for interaction men vs. women).

Mean BMD LS increased in men $+4.2 \mathrm{~g} / \mathrm{cm}^{2}( \pm 4.3 ; p<0.001)$, but not in women +1.5 $\mathrm{g} / \mathrm{cm}^{2}$ ( $\left.\pm 5.6 ; p=0.36\right) ;(\mathrm{p}=0.073$ for interaction)

Figure 2: The change of IGF-1 is positively associated with the change in bone markers during the treatment
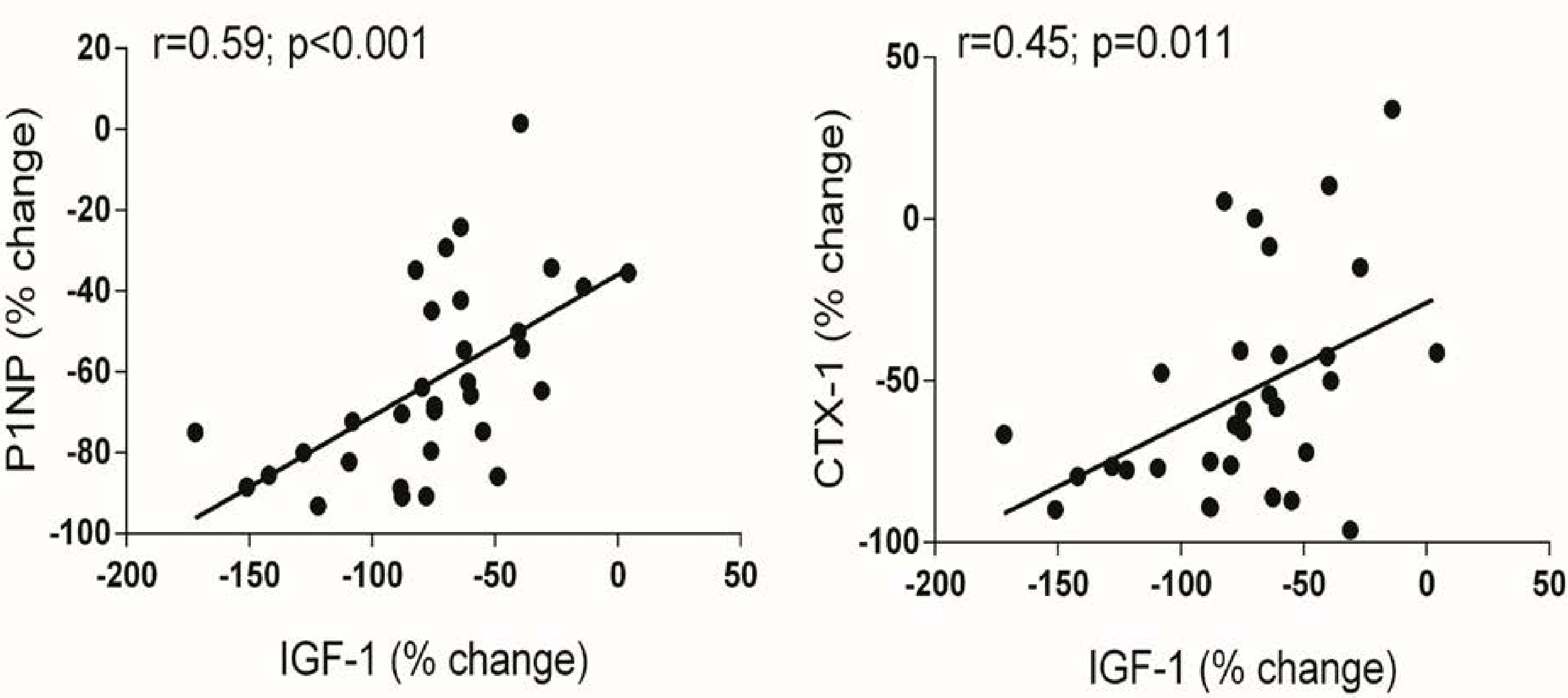

\section{Conclusions}

1) Treatment of acromegaly affects TBS and BMD LS in different and opposite manners; this effect is more pronounced in men.

2) TBS decreased significantly and unexpectedly independent of BMD and bone turnover markers.

3) The reduction of bone turnover markers determined the increase in BMD.

4) TBS, independently of BMD, might reflect alterations in trabecular bone architecture related to the biomechanical properties and explain the increased fracture risk in patients with acromegaly. 